\title{
Quality-Dependent Fusion System using No-reference Image Quality Metrics for Multimodal Biometrics
}

\author{
Pravin G. Gawande ${ }^{1}$, Ashok M. Sapkal ${ }^{2}$ \\ ${ }^{1,2}$ Department of Electronics and Telecommunication Engineering,Government College of Engineering, \\ Shivaji Nagar, Pune, Maharashtra, 411005, India
}

\begin{tabular}{l} 
Article Info \\
\hline Article history: \\
Received Jan $12^{\text {th }}, 2018$ \\
Revised Apr $20^{\text {th }}, 2018$ \\
Accepted Jun $26^{\text {th }}, 2018$ \\
\hline
\end{tabular}

\section{Keyword:}

Quality measures; quality metrics; no-reference; multimodal biometrics; iris; fingerprint; palmprint

\begin{abstract}
Biometric acquired and processed data quality is the prime influences, which will affect the performance of the whole biometric system. Hence, aforementioned is essential to control the quality of acquired data to devise a suitable biometric system. This paper presents a robust multimodal biometric system using quality dependent expert fusion system. We Presents work, on a novel quality assessment metrics for Fingerprint, Palmprint, and Iris. The originality of this work contributing with blind image quality measures. The projected quality metrics associates with two type of quality measure a) Image-based quality as well as b) pattern-based. We have explore and comprehend the associated various quality assessment in the biometrics. Benefits of the proposed quality matric have been illustrates on six benchmark database. The performance of the proposed quality measures demonstrates on multimodal biometric system is evaluated on a public dataset and demonstrating its recognition accuracy with respect to EER. Result shows the efficiency of detecting the kind of alterations. Kolmogorov-Smirnov (KS) test statistics shows 0.84 to 0.94 outperformed as compared to NFIQ.
\end{abstract}

\section{Corresponding Author:}

Pravin G. Gawande,

Department of Electronics and Telecommunication Engineering,Government College of Engineering,

Shivaji Nagar, Pune,Maharashtra, 411005, India

pravingawande3210@gmail.com

\section{Introduction}

Technique of confirming an individuality privilege using individual's physical as well as behavioural appearances is the confirmation of the biometrics. Performance of biometric recognition system deeply effects due to major problem in the deployment of the various errors characterized by biometric raw data during the verification. The sources of errors are such as variations in human characteristics, environmental factors and cross device matching One important way of addressing errors is the make use of quality measures, which we define as information that helps to review the possibility of truthfulness of biometric verification decision.

A current study shows that the performance of the biometrics system decreases due to the unfavourable effects of the corrupting features from the above stated errors in a biometrics system. Study shows that to avoid the effect of these errors there is need to explore the multimodal biometrics (MB). In MB system that the noise will disturbing individual modality will not having any influence on the other biometrics modalities. 
Overall Performance of the system depends on the quality of the acquired data, Fingerprint Verification Competition (FVC) series shows the impact of quality on performance of the system (R Cappelli et al., 2006). There result shows that for the best matching algorithm equal error rate (EER) increased for 0.97 in FVC2000 and FVC2002 to 2.116 in FVC2004 and FVC2006 database. Specifically database used FVC2004 and FVC2006 are further challenging as the uncertainties are purposely introduced. Therefore it is essential to control quality of the selected biometric data in enrolment as well as in verification steps by removing the poor quality samples.

There are two major components are worthwhile to accomplish for capturing various corrupting factors:

\section{- Useful information of quality measures and}

- A useful fusion method able to capture quality trials and match scores as input in class to build a most favorable of agree to take/throw away choice. This technique of fusion is acknowledged as qualitydependent fusion.

In the proposed work, we aim to intend a most favourable best quality focussed fusion technique using image based and pattern based quality measures using scale invariant future transformation (SIFT) keypoint. Which will work efficiently on a given quality set. Advantage of the presented metric can be used for multimodal system.

We have reviewed various quality assessment and quality-dependent fusion techniques which is found in different works has been informed. Outline of the paper is given as, Section 2 Associated work on quality assessment. Section 3 Design of Quality Assessment metrics, Section 4 investigational outcomes performed on benchmark database for Fingerprint, Palmprint and Iris, Section 5 comparison with NFIQ metric of fingerprint. The proposed work ends with section 7 conclusion, followed by references.

\section{Associated work in Quality Assessment and proposed work}

Detail work carried out so far in the area of image quality assessment for multimodal biometric field with existing image based quality metrics and the proposed work for quality assessment in multimodal biometrics is stated here.

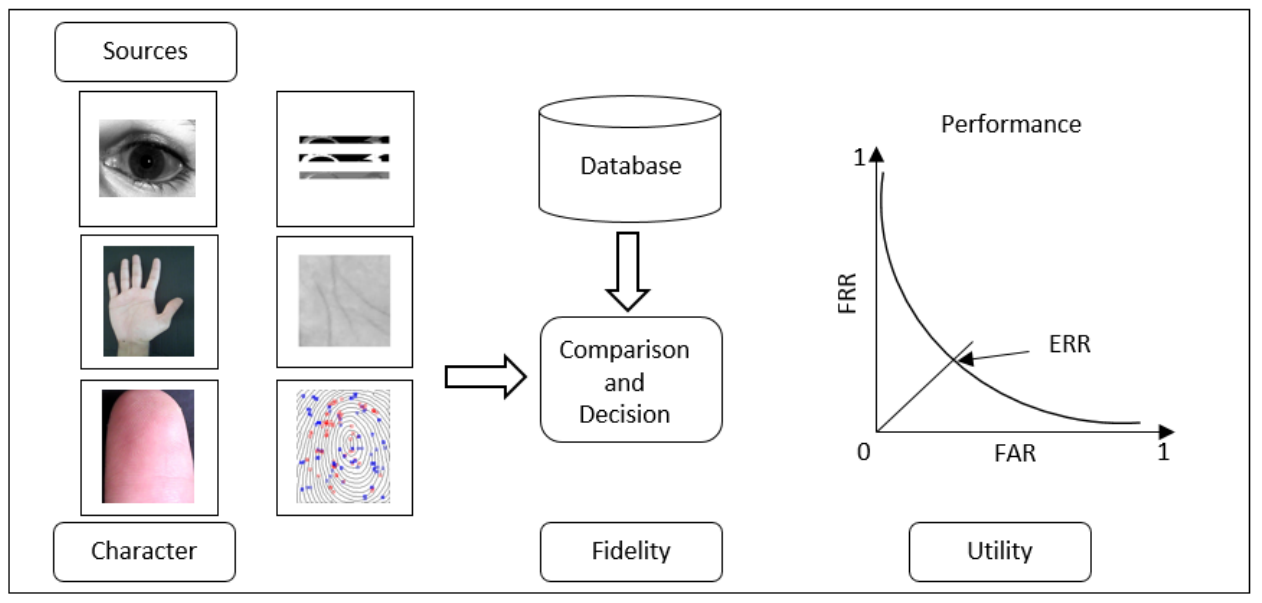

Figure 1. Biometric data quality assessment

\section{1 $\quad$ Associated work in quality assessment metrics}

ISO/IES 29794-1(2009) presents the quality assessment of raw biometrics with different facets, where three significant aspects of quality are acknowledged, these are illustrated in Figure 1.

- Character: Sample in which quality attributes to the physical aspects of the basis since the biometric test being obtained. E.g., a scratched finger has a deprived character.

- Fidelity: Test model in which quality will reviews the relationship among the biometric test with its input.

E.g., a device having low resolution outcomes in a lesser fidelity trial. 
- Utility: Expected influence of each specific trial with complete performance of the whole biometric system and which has been reliant equally with character and the fidelity of the sample.

Shen L., Kot A., Koo W. (2001), used Gabor filters to recognize good quality slabs of ridges and valley pattern and this method mainly used to estimate the quality of fingerprint image precisely. Lim et al. (2002), Chen et al. (2005), Alonso-Fernandez et al. (2007) presents quality metrics for fingerprint that are mainly classifies local features, global features of the image. They presented the efficiency of predicting the quality of fingerprint. But these procedures are dependent on modality and cannot be used for other kind of modalities. National Institute of standard Technology (NIST) Fingerprint Image Quality metric (NFIQ) presented example using these quality metrics and these are only dedicated for fingerprint quality evaluation.

Krichen et al. (2007), Presents Gaussian Mixture Model (GMM) based statistical quality measures, and compared quality measures with standard methods based on Fourier Transforms and wavelets. They verified the performance of the system decreases due to two types of variations used such as occlusions and blurring. Experimental results shows significant improvement in performance of the system using GMM. Chaskar et al. (2012) estimation procedure for nine quality aspects of iris images. Various papers have presented the methods for quality measures. However, these methods are used only for Iris image.

Q He et al. (2008) developed method which is categorized in three computation levels for biometric sample quality i.e. levels are database quality, quality of class, and quality of image. This method is based on matching score distribution of genuine as well as impostor. The drawback of this developed method i.e. it cannot be used for single capture of the modality directly.

Guerrache F. et al. (2016) propose innovative method for computing palmprint captured image quality with respect to illumination, it will discard the palmprint having poor illumination by integrating to the system and to make new session of acquisition.

To achieve better accuracy in biometric system confirmation of the quality of biometric images captures from the sources are essential. From the rigorous survey we found that most of present quality metrics are dependent on modality and matching methods as well as all research activities are towards performance side. In order to get achieve genuine score distribution data it will have need of a large number of trials of the same modality. Therefore the contribution of this paper is to define quality vector which is self-determining and which can be independent on referred matching process to improve the overall performance of the system.

\subsection{General scheme of proposed work}

Figure 2 demonstrates two type of information one image based criteria and another pattern based criteria are used for maintaining quality information. For predicting the quality of the target biometric information SVM multi-class support classification learner process is performed.

\section{Development of quality assessment metric}

Development of quality assessment metric is designed using image quality based biometrics data.

The projected image quality based assessment (IQA) metrics is defined as:

$$
Q=\frac{1}{A} \sum_{i=1}^{N} \alpha_{i} \cdot C_{i}
$$

Where number of the retained criteria is $N$ for $C i ; i=1: N$. Where A is normalisation constant, and $i$ represents the weights we used for optimization. 


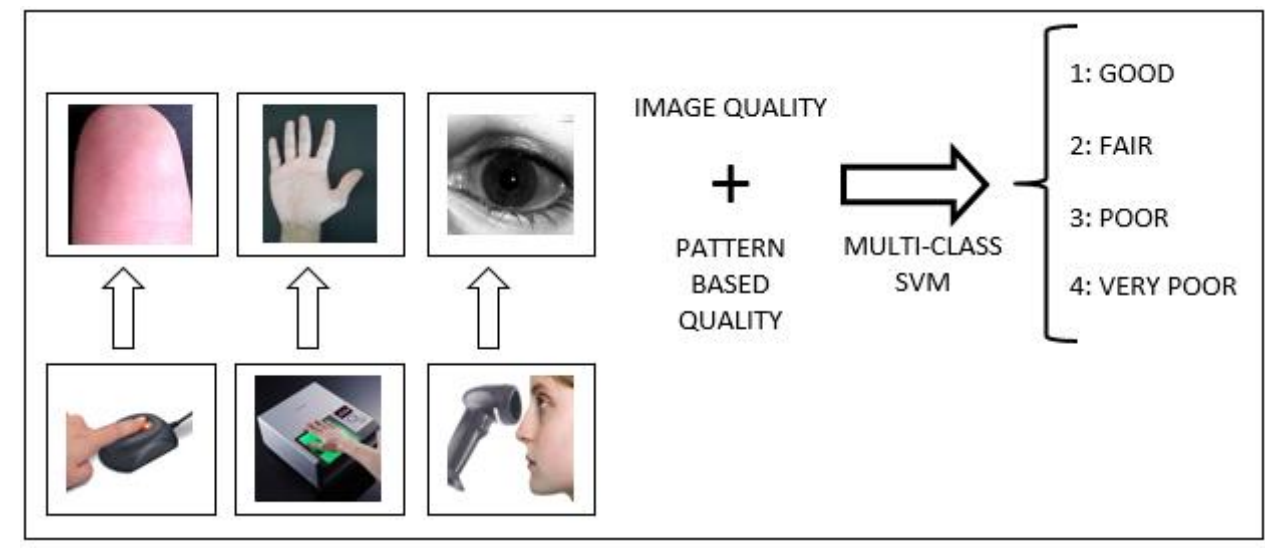

Figure 2. Proposed quality metrics structure

\subsection{Image quality with No-reference}

Quality assessment of the image (IQA) is broadly used to confirm the process applied for digital captured images. IQA separated with three classes: 1) Full-Reference (FR-IAQ), in which output is matched by original image having perfect quality; 2) Reduced -Reference (RR-IAQ); and 3) No-Reference (NR-IAQ). NR-IQA having three approaches: a) Distortion-specific, b) Training-based and c) Natural scene statistics (NSS). We have consider BLIINDS-II (BLind Image Integrity Notator using DCT Statistics) by Saad et al. (2011), represents non-distortion method and extracted features from the DCT-domain. These typical parameters transforms into features. To predict the quality score we have used Multi-class SVM.

Four features are defined from the BLIINDS-II index which is based on DCT (2011).

- Contrast feature (u1)

$$
C^{k}(x)=\frac{1}{N} \sum_{i=1}^{N} \frac{x_{A C}^{i}}{x_{D C}}
$$

$\mathrm{N}$ : patch size, $x_{D C}$ is the DC coefficient and

Set $\left\{x_{A C}^{i} \mid i=1: N\right\}$ are representing AC coefficients.

Contrast value $v_{1}$ is the averaging of all the contrast score on the global image given as:

$$
v_{1}=\frac{1}{M} \sum_{i=1}^{M} c^{i}(x)
$$

$M$ : number of local patches.

- Structural features (U2)

On k patches local DCT frequency coefficients are computed to derive structural features, it is based on DCT histogram of statistical traits where the DC coefficients are ignored. To measure this statistical traits its kurtosis is computed.

$$
K^{k}\left(x_{A C}\right)=\frac{E\left(x_{A C}-\mu\right)^{4}}{\sigma^{4}}
$$

Mean and standard deviation of $x_{A C}$ are combined together and average of all are used to compute kurtosis value $v_{2}$ on the global image.

- Anisotropy orientation (u3 and u4) 
Renyi entropy is the anisotropy measure is computed on DCT along with four diverse directions $\theta=0^{\circ}, 45^{\circ}, 90^{\circ}$, $135^{\circ}$ on image patches. Each patch of DCT is then normalized with the form:

$$
\bar{P}_{\theta}[k, j]=\frac{P_{\theta}[k, j]^{2}}{\sum_{j=1}^{N} P_{\theta}[k, j]^{2}}
$$

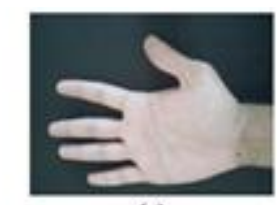

(a)

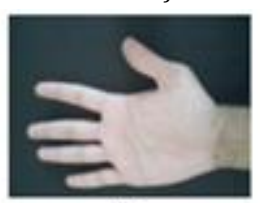

(b)

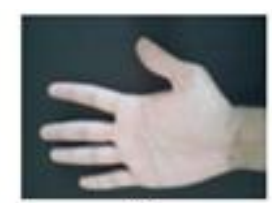

(c)

BLIINDS index on samples of Palmprint database. From (a) to (d), reference image to its noisy images. 29, 27, 26.6 and 26. As categorized as GOOD, FAIR, POOR and VERY POOR

Figure 3. Samples of palmprint database on BLIINDS index.

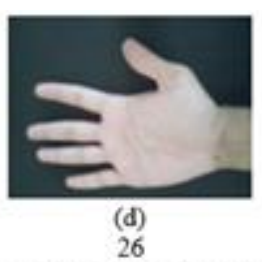

Where size of orientation of $\mathrm{k}^{\text {th }}$ patch is represented as $\mathrm{N}$.

Renyi entropy $R_{\theta}^{k}$ is computed as:

$$
R_{\theta}^{k}=\frac{1}{1-\beta} \log _{2}\left(\sum_{j=1}^{N} \bar{P}_{\theta}[k, j]^{\beta}\right)
$$

Where $\beta>1$.

Lastly, anisotropy measures $v 3$ and $v 4$ are defined as:

$$
\begin{aligned}
& v_{3}=\operatorname{var}\left(E\left(R_{\theta}^{k}\right)\right) \\
& v_{4}=\max \left(E\left(R_{\theta}^{k}\right)\right), \forall k, \forall \theta
\end{aligned}
$$

Final global score is computed as:

$$
\text { BLIINDS }=\prod_{i=1}^{L} v_{1}^{\alpha_{1}^{i}} v_{2}^{\alpha_{2}^{i}} v_{3}^{\alpha_{3}^{i}} v_{4}^{\alpha_{4}^{i}}
$$

Figure 3 represents the predicted quality score using BLIIND index. As the image degraded stronger quality index is decreases.

Image Quality with Pattern-Based

We have apply this method since the main features defined by this is steady way where in region of image having important information. Statistical measures of keypoint features are used which is generated by using Pattern-based quality criteria. This is extensively used method for biometric and object recognition issues.

Several methods are exist in the literatures, like scale-invariant feature transform (SIFT), and speed up robust features (SURF). From the survey, shows that SIFT algorithm outperformed as compared to other methods as given by Mikolajczyk et. al (2005). SIFT algorithm also effectively apply in biometric recognition for various modalities like fingerprint, iris, palmprint and face.

Four major stages in SIFT algorithm that are: scalespace extrema detection, keypoint localization, orientation assignment, and keypoint descriptor. Examples of detected SIFT keypoints are shown in Figure 4. 

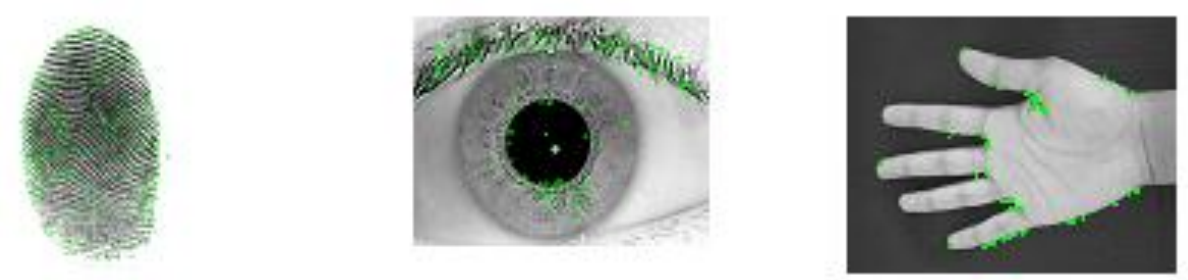

Figure 4. An example of SIFT keypoint detected

\subsection{SVM-based classification}

Support Vector Machine (SVM) used for prediction of sample quality for two methods proposed that are image quality and pattern-based quality. SVM-based techniques have been used due to the high classification rates and high realization and high generalization.

\section{Experimental results}

Proposed six synthetic alteration methods in designed quality metric is to perceive realistic accuracy which is affected in matching systems. Figure 5 illustrates the prediction method using alteration of the input image. Matching module verifies the proposed images as good, fair, poor or else very poor class.

\subsection{Database and Alteration Procedure}

Six standard databases are used and introducing three types of alterations for each database (Gaussian Blurring, Motion and Gaussian Noise) and for each type three alteration levels are used.

\subsubsection{Standard databases}

- FVC2002 having three different databases DB1, DB2, and DB3 it is composed of 100 individuals and 8 samples per subject (2006) sample images are shown in figure 6.

- IITD Iris Image Database version 1.0, it composed of 224 subjects, having five iris images from left and five iris images from right comprising of 16 males and 48 females (2010) sample images are shown in figure 7.

- Polytechnic University Cross-Spectral Iris Images Database, this database consists of total 12,540 iris images, which are acquired with 15 instances from 209 different subjects acquired under simultaneous bi-spectral imaging for both left and right eyes (2017) figure 8,

- And one palmprint database from IITD touchless version 1.0 (2008). Comprised of 235 users and seven samples from each users. Sample images are shown in figure 9. 


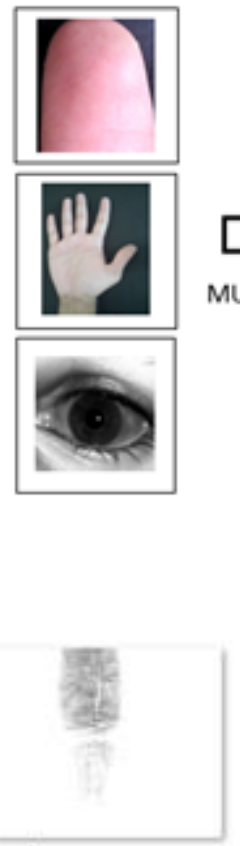

a) $101 \_1$

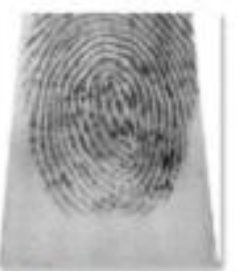

b) $101 \_1$

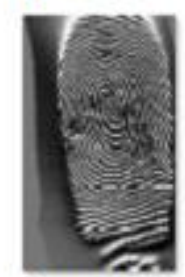

c) $101 \_1$

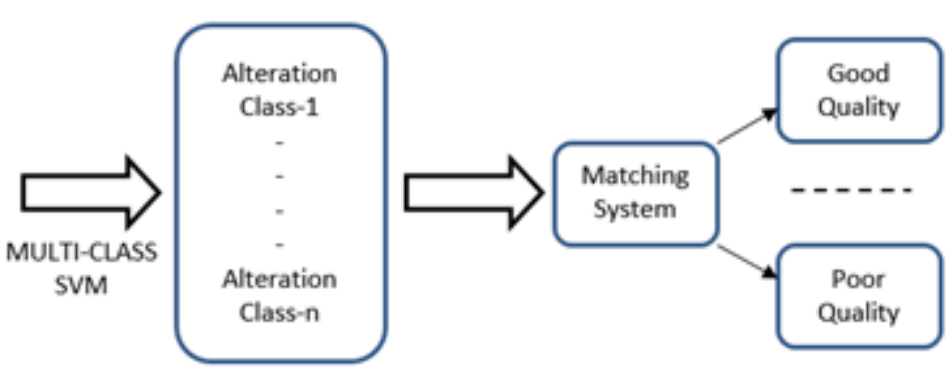

Figure 5. Illustration of presented system

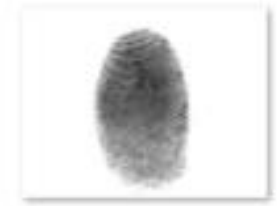

$101 \_2$

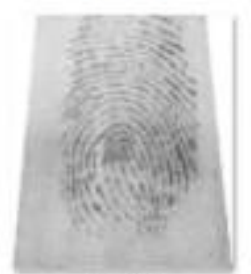

$101 \_2$

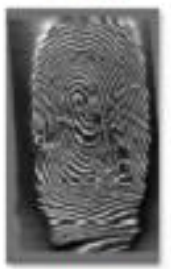

101_2

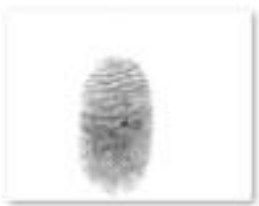

$101 \_3$

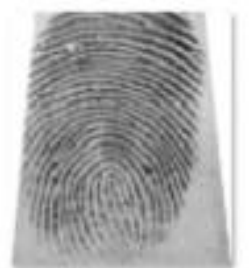

101_3

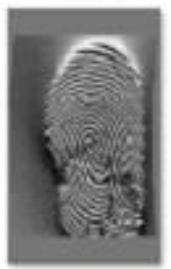

$101 \_3$

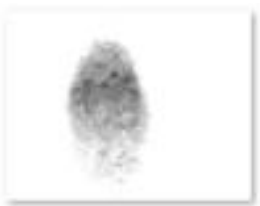

101_4

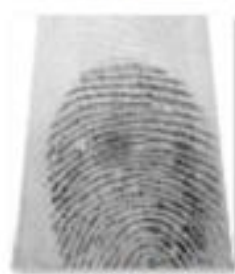

$101 \_4$

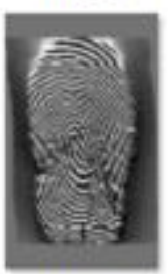

101_4

Figure 6. Sample image from each database FVC2002 a) DB1, b) DB2 and c) DB3:
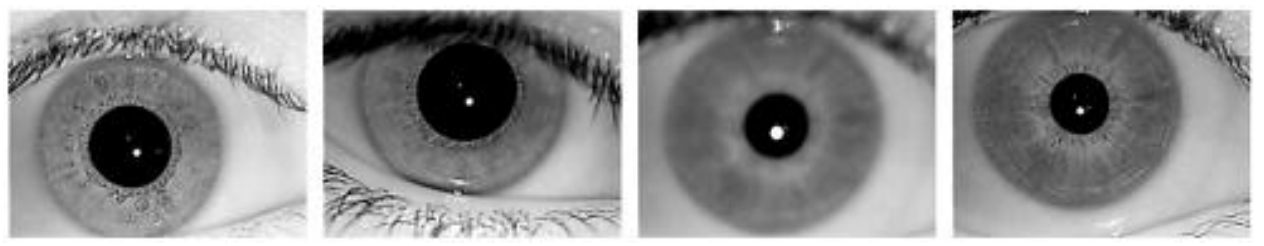

Figure 7. Sample image of iris from IITD database (2010)
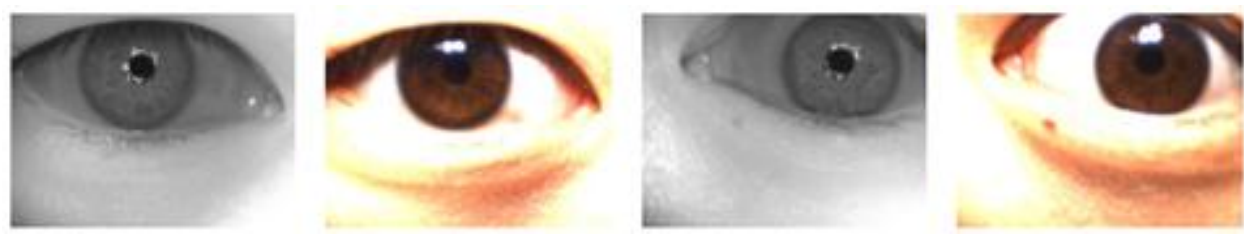

Figure 8. Sample image of iris polytechnic university iris images database (2017) 

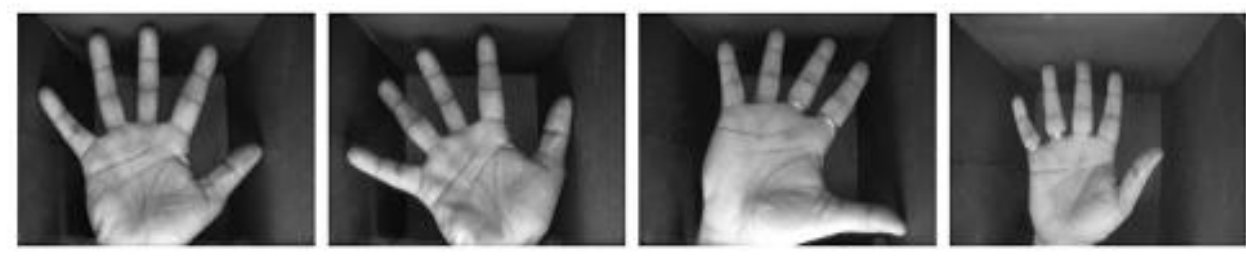

Figure 9. Sample image of touchless palmprint IITD database (2017)

\subsubsection{Alteration procedure for database and classes defined}

We have used two methods for artificial alteration introduced by Muhammad El-Abed et al. (2015) and we introduce one synthetic motion based alteration. So there are three planes of alterations. We have used MATLAB tool for alteration process.

The offered alteration methods are like correct for the period of the attainment with biometric raw information, which will extremely affect the performance of the whole systems. At last, from the various alteration methods we have generated sixty databases: 6 are standard databases and 54 are reformed databases.

Following are the alteration methods are used:

- Gaussian blurring : fspecial('gaussian',hsize,sigma)

- Motion : fspecial ('motion', len, theta)

- Gaussian noise : imnoise(Im, 'gaussian', $n, u)$

Table 1 presents alteration methods using MATLAB for various parameters. These are the input vectors to SVM which will retained quality criteria's i.e. one criteria for image based and four criteria for pattern-based quality and the respective outputs are with ten dissimilar categories specified in Table 2. Figure 10 shows the alterations used for FVC2002 database.

- Original image represents by Class- 1 .

- Alterations levels are represented by Class -2 to Class -10 .

Table 1. Alteration methods using MATLAB for various parameters

\begin{tabular}{|c|c|c|c|c|c|}
\hline \multirow{2}{*}{ Nature } & \multirow{2}{*}{ Technique use for alteration } & \multicolumn{3}{|c|}{ Level } & \multirow{2}{*}{$\begin{array}{c}\text { Number of } \\
\text { Images used } \\
\text { from each } \\
\text { modality }\end{array}$} \\
\hline & & 1 & 2 & 3 & \\
\hline $\begin{array}{l}\text { Gaussian } \\
\text { blurring }\end{array}$ & Fspecial ('gaussian',hsize,sigma) & $\sigma=1$ & $\sigma=2$ & $\sigma=3$ & 50 \\
\hline $\begin{array}{l}\text { Motion } \\
\text { blurring }\end{array}$ & $\begin{array}{l}\text { fspecial } \\
\text { ('motion',len,theta) }\end{array}$ & $\Theta=30$ & $\Theta=45$ & $\Theta=50$ & 50 \\
\hline $\begin{array}{l}\text { Gaussian } \\
\text { noise }\end{array}$ & $\begin{array}{l}\text { Imnoise } \\
\text { (Im,'gaussian',0.01,u) }\end{array}$ & $\mathrm{u}=0.004$ & $\mathrm{u}=0.02$ & $\mathrm{u}=0.019$ & 50 \\
\hline
\end{tabular}

Table 2. SVM class description

\begin{tabular}{lll}
\hline Classes & Alteration method used & Levels of Alteration \\
\hline 1 & Original image & $\mathrm{x}$ \\
2,3 and 4 & Gaussian blurring & 1,2 and 3 \\
5,6 , and 7 & Motion blurring & 1,2 and 3 \\
8,9 and 10 & Gaussian noise & 1,2 and 3 \\
\hline
\end{tabular}



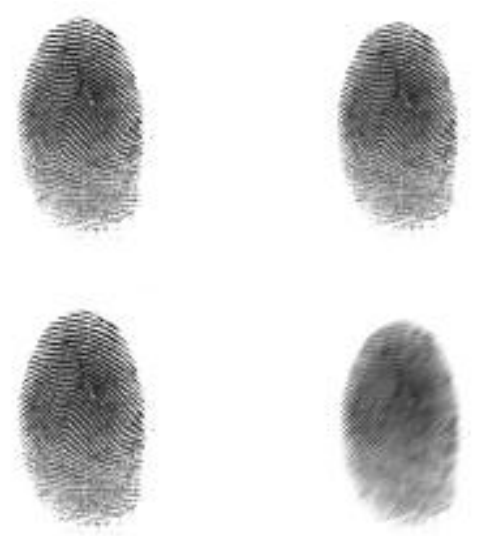

(a)
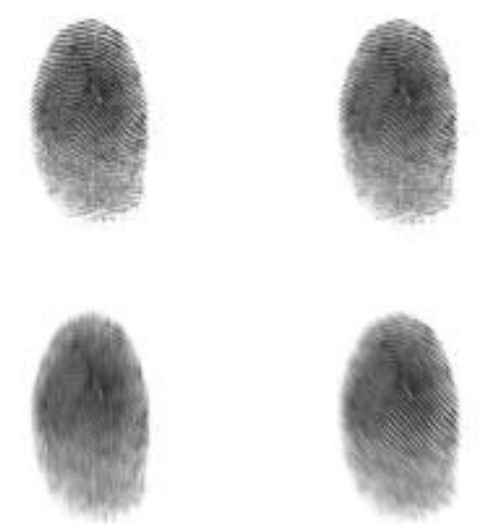

(b)
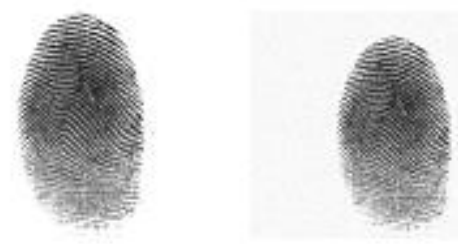
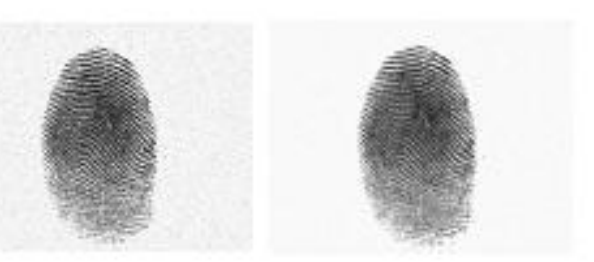

(c)

Figure 10. Reference image from FVC2002 DB1 for alteration. From left side, reference and modification stages 1, 2 , and 3. (a)Gaussian Blurring, (b) Motion blurring, and (c) Gaussian noise alteration.

\subsubsection{Matching algorithm for Biometric}

To validate the proposed quality metrics of biometrics matching algorithms are used. Algorithm is used for biometric matching is based on SIFT (2004). The confirmation among two images im1 and im2 agrees to the likeness of sets of two features X (im1) as well as X (im2). Matching method we are using improved form of a decision criterion which is suggested by Lowe (2004). Specified two key-points $x \in X$ (im1) and $y \in X$ (im2), assume that $x$ is related to $y$ if:

$$
d(x, y)=\min _{\left[z \in X\left(i m_{2}\right)\right]} d(x, z) \text { and } d(x, z) \text { and } d(x, y) \leq C d(x, y !)
$$

$\mathrm{C}$ represents subjective threshold,

Euclidean distance represented by $\mathrm{d}(\cdot, \cdot)$ among the SIFT based descriptors and y signifies point of X (im2) for which distance for $\mathrm{x}$ is marginal but larger than $\mathrm{d}(\mathrm{x}, \mathrm{y})$ :

$$
d(x, y !)=\min _{\left[z \in X\left(i m_{2}\right), d(x, y)>d(x, y)\right]} d(x, z)
$$

\subsection{Validation process}

Performance of the system should be predicted by the biometric quality metric, Patrick Grother, Elham Tabassi (2007). From biometric raw information we have created quality metrics and generated a class related to error rates related with the overall biometric performance (2009). As EER will be lower system will be considered as more accurate. For confirming the validation of the projected quality metric, we carry on as follows:

- Alteration and performance of quality criteria: Validation process is the first step to check the robustness of the behavior of the five quality criteria detecting by using various alteration methods: Gaussian blurring alteration. Motion blurring and Gaussian noise. 
- Multi-class SVM learning: Three multi-class SVM types we learn for fingerprint databases, we have used images from the three standard databases for fingerprint. For the iris database two multi-class SVM, and for palmprint database we learn one SVM model.

- EER values for quality set: EER values are calculated using our predicted labels metric. The performance of this system is evaluate to check how fine this quality information and used to calculate performance of the system by the using respective quality set. As further images are degraded performance of the complete arrangement will be decreases.

\section{$4.3 \quad$ Results}

Pearson's correlation coefficient criteria has been used to check the robustness of the measures in perceiving alterations in the captured modalities. Equation 9 and 10 shows Pearson's coefficient among two different variables. Covariance is given as:

$$
\begin{gathered}
\rho(A, B)=\frac{1}{N-1} \sum_{i=1}^{N}\left(\overline{\frac{A_{l}-\mu_{A}}{\sigma_{A}}}\right)\left(\frac{B_{i}-\mu_{B}}{\sigma_{B}}\right) \\
\rho(A, B)=\frac{\operatorname{cov}(A, B)}{\sigma_{A} \sigma_{B}}
\end{gathered}
$$

Computing the correlation of the three types of alteration for variable $p$ the defined criteria are:

- $A_{p}=\left\{A_{p k} \mid k=1: 4\right\}$

For original database $A_{p 1}$ is a group with values for criterion $p$, and for reformed database $\left(A_{p 2}, A_{p 3}\right.$ $A_{p 4}$ ) represents groups with values for criterion $p$ representing levels I, II, and III.

- Modification levels are denoted by $B$ (1: is for standard databases; 2, 3 and 4: represents modified databases stages I, II, and III). More precisely,

$$
\begin{aligned}
B=\{b k \mid b k & =1 \text { for } k=1: N, \\
b k & =2 \text { for } k=N+1: 2 N, \\
b k & =3 \text { for } k=2 N+1: 3 N \\
\text { and } b k=4 \text { for } k & =3 N+1: 4 N\}
\end{aligned}
$$

Reference database size represented by $\mathrm{N}$.

Table 3 shows five dimensional vector $V$, predictor for quality of the sample image.

Figure 11 shows five dimensional vector for image based and pattern based criteria relevant in perceiving the following types of alterations: (a) Gaussian alteration, (b) Motion blurring alteration, and (c) Gaussian noise alteration. BLIINDS shows to be competent correlation coefficient more than 0.7 in detecting Gaussian noise as well as blurring alterations.

Table 3. The vector $V$ predictor for biometric quality

\begin{tabular}{llllll}
\hline Criterion & $\begin{array}{l}\text { Image-based } \\
\text { quality criteria }\end{array}$ & Pattern-based quality criteria \\
\hline Vector $\boldsymbol{V}$ & BLIINDS & Keypoints & $D C$ coefficient & $\begin{array}{l}\text { Mean }(\mu) \\
\text { scales }\end{array}$ & $\begin{array}{l}\text { of } \\
\text { deviation } \\
\text { of scales }\end{array}$ \\
\hline
\end{tabular}




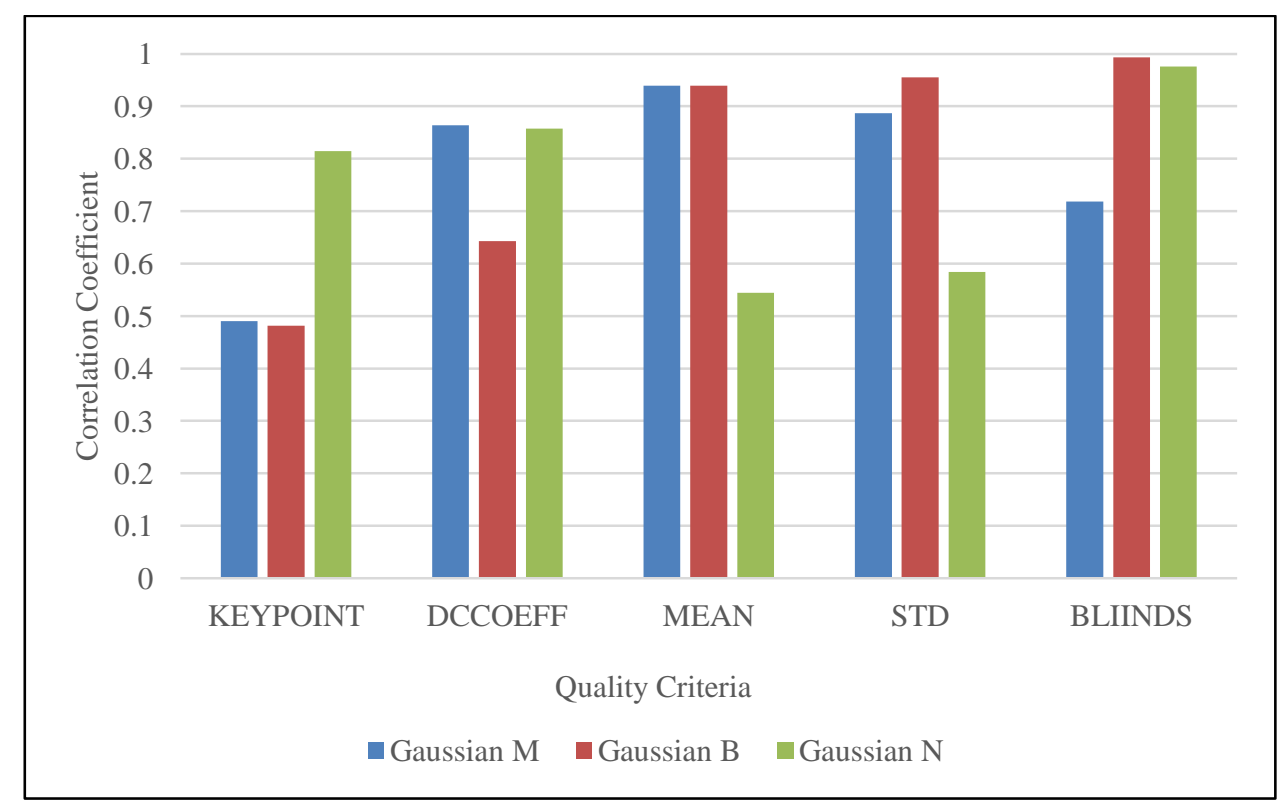

Figure 11. Correlation for the projected criteria and the alterations for three fingerprint databases.

\subsection{Model - Multi-class SVM}

Eight multiclass SVM models are learned: four for fingerprint, three for iris and one for palmprint database. Table 4 presents the accuracy and the efficiency of the projected quality metric in predicting the artificial modifications of data, with a valuable 4-class and 10-class classification accuracy using multiclass SVM model (going from $82.9 \%$ to $99.3 \%$ ).

Table 4. Ten-class classification Accuracy (\%) by Multiclass SVM for $\mathrm{SVM}_{\text {each }}$ and $\mathrm{SVM}_{\text {all }}$

\begin{tabular}{lcc}
\hline \multirow{2}{*}{ DATABASE } & \multicolumn{2}{c}{ SVM Accuracy in \% } \\
\cline { 2 - 3 } & SVM $_{\text {each (4-CLASS) }}$ & SVM $_{\text {all (10-CLASS) }}$ \\
\hline FVC2002 DB1 & 99.1 & 82.9 \\
FVC2002 DB2 & 99.3 & 93.9 \\
FVC2002 DB3 & 98.8 & 94.4 \\
IITD IRIS & 96.3 & 94.5 \\
PolyU IRIS & 97.5 & 93.5 \\
IITD Palmprint & 97.5 & 90 \\
\hline
\end{tabular}

Table 5. Category of quality for 10 class

\begin{tabular}{lcc}
\hline Set of Quality & $\begin{array}{c}\text { 10 Quality class } \\
(\text { Predicted by SVM })\end{array}$ & Report \\
\hline I & 1 & GOOD \\
II & 2,5 and 8 & FAIR \\
III & 3,6 and 9 & POOR \\
IV & 4,7 and 10 & VERY POOR \\
\hline
\end{tabular}




\subsubsection{Definitions of Quality sets}

Robustness of the proposed quality metrics is to quantify for predicting the system performance we have to define the quality sets. Robustness of the system has been tested against the three alterations levels. The EER values calculated using the first enrolled image as a reference and the rest altered images for the test. Figure 13 shows that all the introduced alterations have an impact on overall performance of the used authentication matching algorithm. Table 5 defines the quality sets for used matching algorithm.

Figure 12 shows EER values of each quality set for the used three fingerprint dataset computed using three multiclass SVM i.e.one multiclass SVM per database. According to Patrick Grother, Elham Tabassi (2007) we have calculated EER value for each quality set which was predicted by multiclass SVM model. The propose metrics has shown the efficiency for predicting the matched algorithm among the datasets. It shows that the more EER is increased as more the images are altered.
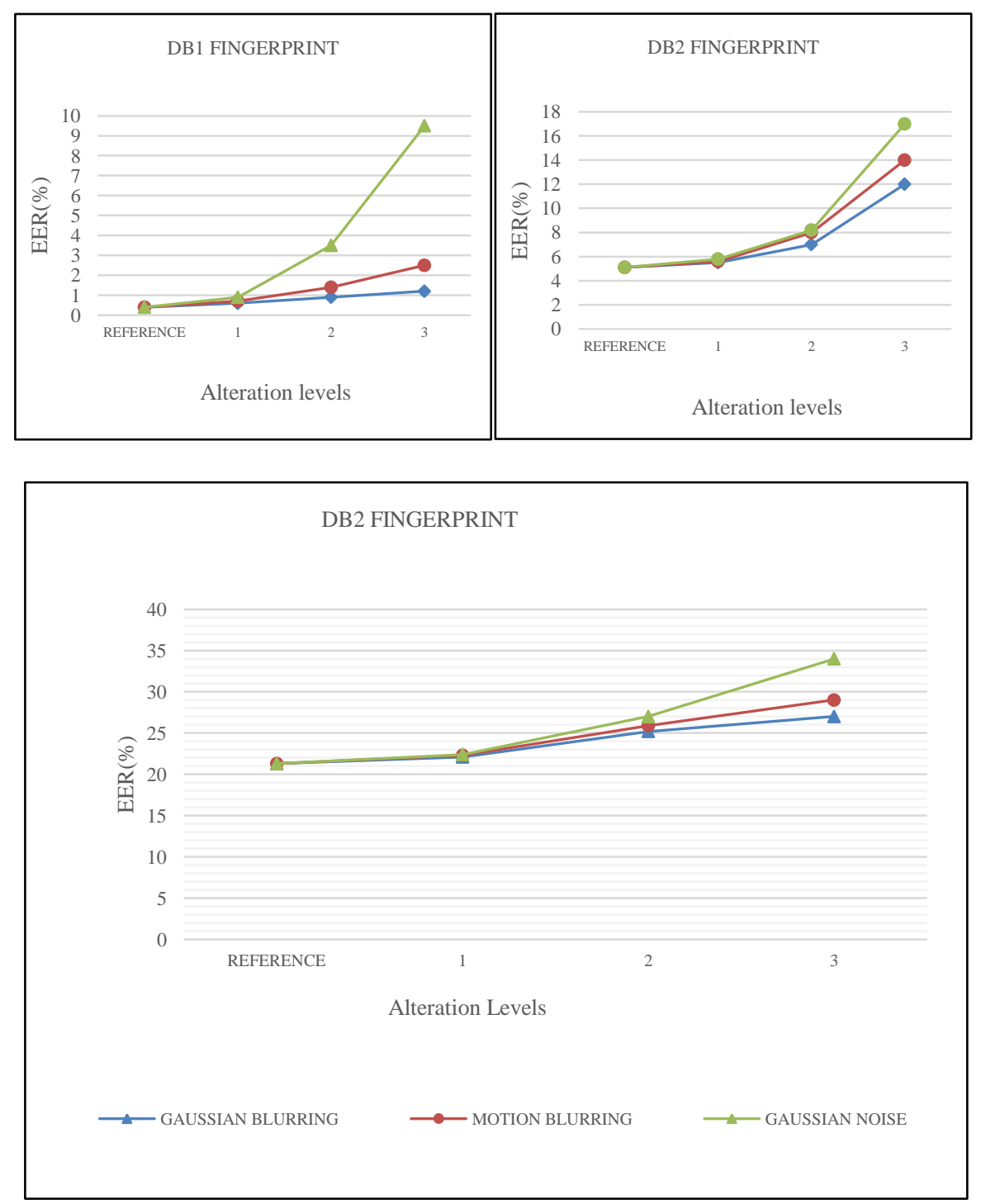

Figure 12. Impact of alteration levels on performance of overall system among the three fingerprint dataset 


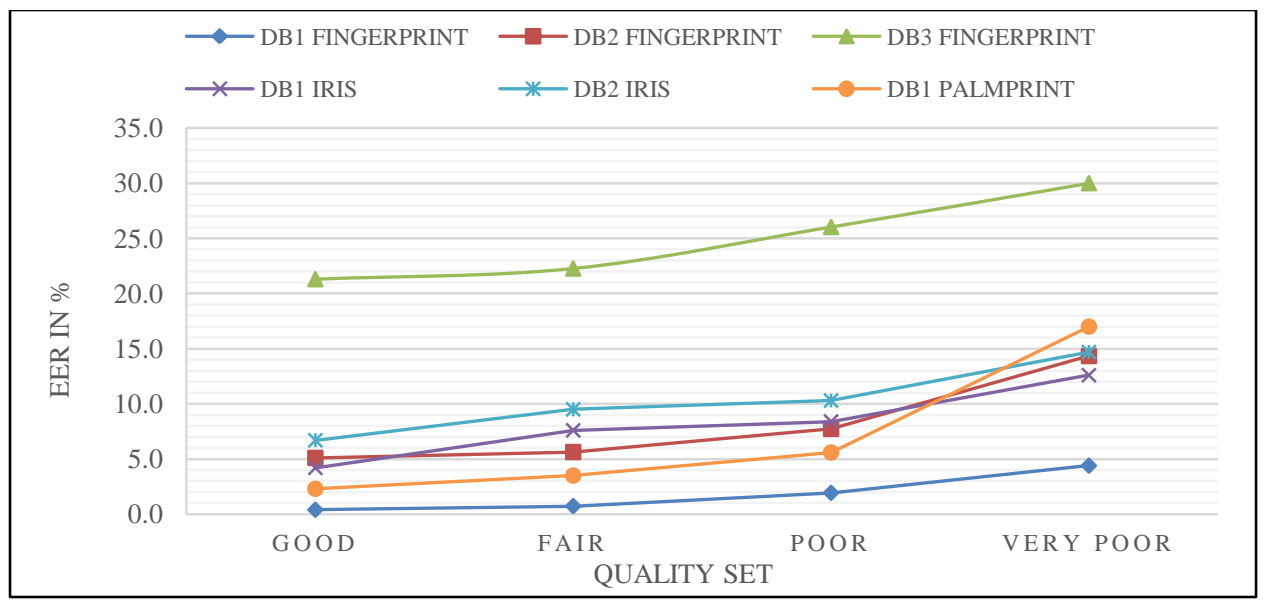

Figure 13. EER in \% of each quality set for biometric databases fingerprint, iris and palmprint.

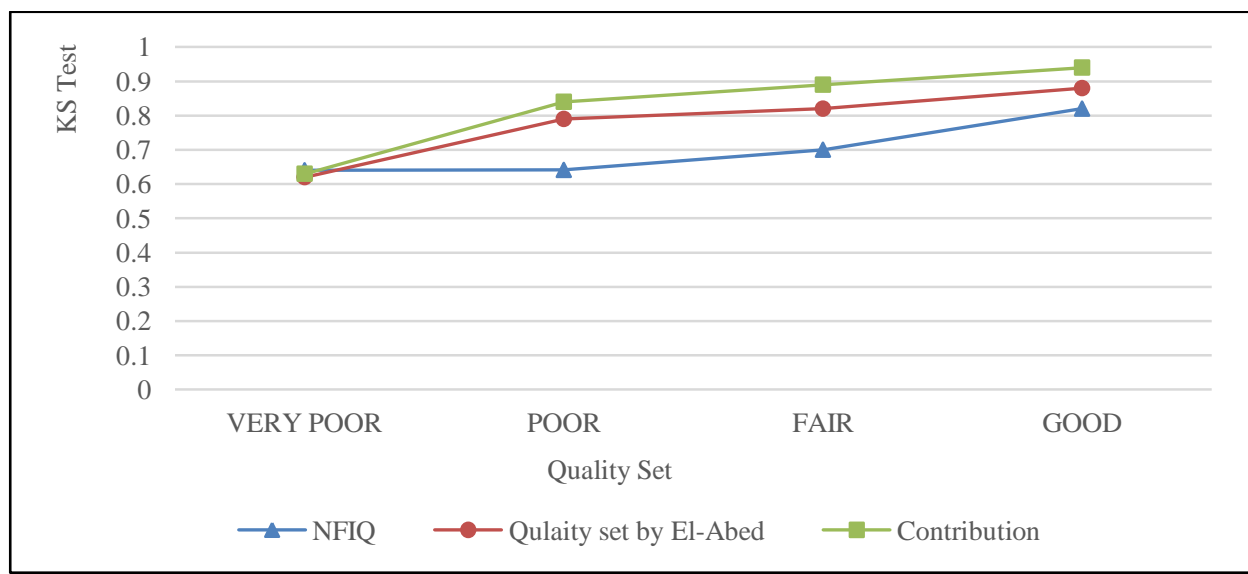

Figure 14. Comparison study of proposed metric with NFIQ and Quality metrics proposed by El-Abed using Kolmogorov-Smirnov (KS) test statistic.

\section{Comparison study with standard metrics defined by NFIQ}

In this section we have presents a comparison study to show the efficiency of the proposed quality metrics with NFIQ metrics proposed by NIST and the quality metrics defined by El-Abed (2015). For the fingerprint NIST is the most cited work. Five quality levels are provided by NFIQ i.e. NFIQ=1 shows superior quality sample and NFIQ=5 indicated deprived or poor quality samples. As we have proposed quality set with four levels, so we have to considered the fourth and fifth levels of NFIQ as very poor quality set. Kolmogorov-Smirnov (KS) test (1990) is used to match the projected metrics with NFIQ, this method has been proposed by Grother and Tabassi (2007). KS test should be expected large for better quality samples.

Figure 14 shows the KS test statistic values compared with quality metrics NFIQ, quality metrics by El-Abed and the proposed quality metric for three quality sets i.e. poor, fair and good. Figure 14 illustrates that for the proposed metrics KS statistics ranges from 0.84 to 0.94 outperformed as compared to NFIQ (0.641 to 0.82$)$ metric and quality metrics by EI-Abed (0.79 to 0.88$)$.

\section{Conclusion}

In biometrics system quality assessment of raw data is the important factor during the enrollment step. This information may be used to improve the performance of the system. Few papers have presented the comparison on the performance. We proposed image-based quality assessment metrics of raw biometric information using image and pattern based quality. This metrics is used to several kind of modalities and independent on used matching system. We have used six 
publically available biometrics database (Fingerprint, iris and Palmprint). Result shows the efficiency of detecting the three kind of synthetic alterations such as Gaussian blurring, Motion blurring and Gaussian noise. We have also compared the proposed metrics with NFIQ using biometric matching algorithm proposed by NIST as well as with the quality metrics of El-Abed.

\section{References}

ISO/IEC 29794-1, (2009), Biometric Quality Framework Standard, First Ed. JTC1/SC37/Working Group 3.

Shen L., Kot A., Koo W. (2001) 'Quality Measures of Fingerprint Images', In: Bigun J., Smeraldi F. (eds) Audio- and Video-Based Biometric Person Authentication. AVBPA 2001. Lecture Notes in Computer Science, vol 2091. Springer, Berlin, Heidelberg, pp. 266-271.

E Lim, X Jiang, W-Y Yau (2002) 'Fingerprint quality and validity analysis', in IEEE International Conference on Image Processing (ICIP). pp. 469-472.

R Cappelli, D Maio, D Maltoni, JL Wayman, AK Jain (2006), 'Performance evaluation of fingerprint verification systems'. IEEE Trans. Pattern Anal. Mach. Intell. (PAMI). 28, pp 3-18

F Alonso-Fernandez, J Fierrez, J Ortega-Garcia, J Gonzalez-Rodriguez, H Fronthaler, K Kollreider, J Bigun, (2007) 'A comparative study of fingerprint image-quality estimation methods', IEEE Transactions on Information Forensics and Security. Volume 2, issue 4, pp 734-743.

Y Chen, SC Dass, AK Jain (2005) 'Fingerprint quality indices for predicting authentication performance', 5th International Conference Audio- and Video-Based Biometric Person Authentication (AVBPA). vol. 3546, pp. 160-170.

$\mathrm{Q}$ He, ZA Sun, TN Tan, Y Zou (2008) 'A hierarchical model for the evaluation of biometric sample quality', International Conference on Pattern Recognition (ICPR). pp. 1-4

E Krichen, S Garcia Salicetti, B Dorizzi, (2007), 'A new probabilistic iris quality measure for comprehensive noise detection', IEEE Third International Conference on Biometrics: Theory, Applications and Systems (BTAS). pp. 1-6.

U M Chaskar, M S Sutaone, N S Shah, T Jaison, (2012) 'Iris image quality assessment for biometric application', IJCSI Int. J. Comput. Sci. Issues. 9, pp. 474-478.

Guerrache F., Haddadou H. (2016),'Palmprint Image Quality Measurement Algorithm'. In Modelling and Implementation of Complex Systems. Lecture Notes in Networks and Systems, vol 1. Springer, Cham, pp. 19-29.

Adams Kong, David Zhang, Mohamed Kamel (2009) 'A survey of palmprint recognition, Pattern Recognition, Volume 42, Issue 7, pp. 1408-1418.

M. Saad, A. C. Bovik, and C. Charrier (2011) 'DCT-statistics model-based blind image quality assessment', in IEEE International Conference on Image Proc. (ICIP), Brussels, Belgium, pp. 3154-3157.

Krystian Mikolajczyk, Cordelia Schmid (2005) 'A performance evaluation of local descriptors', IEEE Transactions on Pattern Analysis and Machine Intelligence, Institute of Electrical and Electronics Engineers, , 27 (10), pp.1615-1630.

El-Abed, M., Charrier, C. \& Rosenberger, C. J Image Video Proc. (2015) 'Quality assessment of image-based biometric Information', EURASIP Journal on Image and Video Processing, 2015: 3. https://doi.org/10.1186/s13640-015-0055-8.

D Maio, D Maltoni, R Cappelli, JL Wayman, AK Jain (2002) 'Fvc2002: Second fingerprint verification competition', in International Conference on Pattern Recognition (ICPR'02), vol. 3 (IEEE Washington, DC, USA), pp. 811-814.

N. Pattabhi Ramaiah and Ajay Kumar (2017) 'Towards more accurate iris recognition using bi-spectral imaging and cross-spectral matching capability', IEEE Transactions on Image Processing, vol. 26, pp. 208-221.

D.G. Lowe (2004) 'Distinctive image features from scale-invariant keypoints', International Journal of Computer Vision. (IJCV). Volume 60, Issue 2, pp. 91-110.

Patrick Grother, Elham Tabassi (2007) 'Performance of biometric quality measures', IEEE Transactions on Pattern Analysis and Machine Intelligence, Volume 29,Issue 4, pp. 531-543.

G Saporta (1990) Probabilit'es, analyse des donn'ees et statistique. Editions Technip, 